\title{
Declarative memory consolidation: Mechanisms acting during human sleep
}

\author{
Steffen Gais and Jan Born ${ }^{1}$ \\ Department of Neuroendocrinology, University of Lübeck, Lübeck 23538, Germany
}

\begin{abstract}
Of late, an increasing number of studies have shown a strong relationship between sleep and memory. Here we summarize a series of our own studies in humans supporting a beneficial influence of slow-wave sleep (SWS) on declarative memory formation, and try to identify some mechanisms that might underlie this influence. Specifically, these experiments show that declarative memory benefits mainly from sleep periods dominated by SWS, whereas there is no consistent benefit of this memory from periods rich in rapid eye movement (REM) sleep. A main mechanism of declarative memory formation is believed to be the reactivation of newly acquired memory representations in hippocampal networks that stimulates a transfer and integration of these representations into neocortical neuronal networks. Consistent with this model, spindle activity and slow oscillation-related EEG coherence increase during early sleep after intense declarative learning in humans, signs that together point toward a neocortical reprocessing of the learned material. In addition, sleep seems to provide an optimal milieu for declarative memory reprocessing and consolidation by reducing cholinergic activation and the cortisol feedback to the hippocampus during SWS.
\end{abstract}

For a long time now, more and more studies have shown that sleep alters the consolidation of memories. The first evidence for this came from studies on declarative memory. These studies showed a better retention of nonsense syllables and short stories over intervals filled with sleep compared with intervals of wakefulness (Jenkins and Dallenbach 1924; Van Ormer 1933; Newman 1939). In recent years procedural memory consolidation has also been found to benefit from sleep. For example, learning of a visual discrimination task or speed in specific sequences of finger tapping were found to be dependent on posttraining sleep intervals (Karni et al. 1994; Fischer et al. 2002; Walker et al. 2002). In fact, certain types of procedural memory develop only if subjects sleep after training (Gais et al. 2000; Stickgold et al. 2000a). Here we concentrate on a number of studies conducted in the authors' laboratory to enlighten some of the mechanisms underlying the enhancing effect of sleep on declarative memory. The studies were all performed in humans, although they rely on concepts derived from observations in animals.

Different sleep stages for memory formation

After the discovery of rapid eye movement (REM) sleep by Aserinsky and Kleitman (1953), most studies were concerned with finding associations between sleep stages and memory consolidation. The prevailing hypothesis-influenced by psychoanalytic theory-was that new memory content would be reprocessed during dreaming, which was thought to occur during REM sleep (Feldman and Dement 1968; Empson and Clarke 1970; Chernik 1972; Lewin and Glaubman 1975). However, these studies, which all used declarative memory tasks, provided very mixed results and were criticized for methodological reasons, leading some researchers to reject a REM sleep-memory hypothesis completely (Vertes and Eastman 2000; Siegel 2001). The method used in these experiments, namely, REM sleep deprivation, was particularly strongly criticized for its nonspecific impairment of cognitive function (Born and Gais 2000).

\footnotetext{
${ }^{1}$ Corresponding author.

E-mail born@kfg.mu-luebeck.de; fax 49-4515003640.

Article and publication are at http://www.learnmem.org/cgi/doi/10.1101/ Im.80504.
}

An experimental design preventing these unspecific effects of REM sleep deprivation was devised by Ekstrand and colleagues (Yaroush et al. 1971). They had subjects learn a pairedassociate list of words before the first or the second half of the night. Then subjects slept for $3 \mathrm{~h}$, and recall was tested after sleep. Because the first half of the night contains high amounts of slow-wave sleep (SWS) and the second half contains high amounts of REM sleep, they were able to compare sleep with different percentages of these sleep stages without disturbing the subjects' sleep. The amounts of non-REM sleep in stages 1 and 2 in the retention sleep periods typically do not differ between the first and second night half in this design. The results pointed for the first time to an importance of SWS for declarative memory (Yaroush et al. 1971; Fowler et al. 1973). The same design that splits nocturnal retention sleep into two halves was built upon in two studies that used procedural memory tasks in addition to declarative tasks (Plihal and Born 1997, 1999a). These studies found a distinct positive influence of the SWS-rich first half of the night on declarative memory consolidation in a paired associate wordlist and a spatial learning task. Sleep from the second half of the night, which is rich in REM sleep, improved nondeclarative memory tasks such as the procedural mirror tracing task and priming processes.

This picture has become more and more complex over the past years with more and more studies examining different tasks and different memory systems. For example, consolidation of declarative memory for highly emotional material was found to benefit additionally from sleep with high amounts of REM sleep (Wagner et al. 2001). This and related studies (Wagner et al. 2002) have led to the suggestion that REM sleep enhances emotional memories. Other studies have indicated that, apart from SWS and REM sleep, transitory sleep stages and particularly the human non-REM sleep stage 2 per se contribute to memory formation, for example, in a simple procedural pursuit rotor task and in cognitive procedural tasks (Smith and MacNeill 1994; Smith and Fazekas 1997). In addition, procedural visual discrimination learning not only depends on REM sleep but also requires a certain amount of preceding SWS (Gais et al. 2000; Stickgold et al. 2000b). 
Together, these results provide good evidence that there are processes of memory consolidation taking place during sleep. However, although declarative memory benefits preferentially from periods in which SWS is predominant and the benefit for nondeclarative types of memory appears to be more related to sleep periods rich in REM sleep, the relationship between individual sleep stages and memory consolidation is probably not that simple. It is more likely that there are different neurophysiological processes at work during the various sleep stages underlying these consolidation phenomena, and that not all of these processes are involved in every form of memory. There are several candidate mechanisms that could account for the changes in declarative memory observed across sleep. These are mainly electrophysiological-, neurotransmitter-, and neuroendocrinerelated mechanisms that are known to play a part in memory function and to exhibit different activity between SWS and REM sleep as well as between sleep and wakefulness.

\section{Reactivation of newly formed memories during sleep}

The covert reactivation of neuronal populations used for encoding the respective materials during prior learning is thought to be a central mechanism for memory consolidation during sleep (Maquet 2001; Stickgold et al. 2001; McNaughton et al. 2003). The reactivation during sleep remains covert because it is not associated with the same or similar subjective experience or overt behaviors as the original activation, suggesting that the underlying neuronal pattern of reactivation also differs in some aspects from the original activation. Evidence for an off-line reactivation has been provided mainly from studies in rats using hippocampus-dependent spatial learning tasks. By using single and multiple unit recordings, it was consistently found that hippocampal activity observed during encoding was replayed during subsequent periods of SWS (Pavlides and Winson 1989; Wilson and McNaughton 1994; Kudrimoti et al. 1999). One study reported a hippocampal replay activity occurring during REM sleep after learning (Louie and Wilson 2001). The replay activity during SWS does not appear to be limited to the hippocampus but extends to other structures, including the thalamus and neocortex (Qin et al. 1997; Ribeiro et al. 2004). In humans, it seems more difficult to reveal signs of a replay of newly acquired memories during sleep. By using positron emission tomography, Maquet et al. (2000) demonstrated in healthy humans a reactivation in the cuneus and the left premotor cortex during REM sleep that followed training on a serial reaction time task. Because performance on this task is thought not to depend on hippocampal function, this finding fits well with the behavioral data discussed above, indicating for hippocampus-independent procedural types of memory overall a greater improvement from sleep periods rich in REM sleep. On the other hand, reactivations observed in rats preferentially during SWS after training on a hippocampus-dependent spatial task agree with our human data, indicating preferential benefits of declarative memory from sleep periods rich in SWS. However, whether and to what extent the signs of reactivation observed during sleep play a functional role in consolidating respective neural memory representations or merely reflect use-dependent phenomena of inert neural activity is presently not clear.

With regard to declarative memory, it has been proposed that the reactivation of hippocampal memory representations during sleep drives a transfer of information to neocortical networks in which it becomes consolidated and integrated into long-term representations residing in neocortical networks (McClelland et al. 1995; Buzsáki 1996; McNaughton et al. 2003). According to this concept, during wakefulness new information enters the hippocampal CA3 region through the entorhinal cor- tex, where it is stored temporarily without disturbing previously acquired memories. During non-REM and SWS, the flow of information is reversed and hippocampal efferents to the neocortex become predominant. In line with this concept, Qin et al. (1997) found signs of coherent neuronal reactivation between hippocampal and neocortical regions and within these regions during SWS following acquisition of a spatial task in rats. Moreover, in the hippocampus the replay of activity during SWS is linked to a sharp wave-ripple pattern of EEG activity. Notably, the sharp wave-ripple pattern has been found to occur in close temporal correlation with sleep oscillatory spindle activity (12$15 \mathrm{~Hz}$ ) in the neocortex, suggesting that spindle activity might be another indicator of hippocampo-neocortical information transfer (Siapas and Wilson 1998; Sirota et al. 2003). Neocortical spindle activity has been considered to be associated with massive calcium influx into pyramidal cells that would via the activation of calcium-sensitive kinases such as CaMKII predispose the cells to the induction of plastic synaptic changes underlying long-term storage (Contreras et al. 1997a; Sejnowski and Destexhe 2000).

\section{Electrophysiological signs: Spindle activity, direct current potentials, and slow oscillations}

Against the background of the outlined reactivation concept for sleep-dependent memory consolidation, the influence of intense learning of a declarative task on the expression of spindles during subsequent intense learning of a hippocampus-dependent declarative memory task (Gais et al. 2002) was studied in healthy humans. In the learning condition, subjects had to learn a list of 336 pairs of unrelated words in the 1 - $h$ period before they went to sleep. In the nonlearning control condition, subjects were presented with a similar list of word-pairs. However, this time they were required to count all letters containing curved lines (e.g., J, $\mathrm{P}, \mathrm{U}$, but not $\mathrm{W}, \mathrm{Y}, \mathrm{K}$ ) in the word displays. Rated task difficulty was comparable for both conditions, but only in the learning condition did subjects semantically process the words for explicit learning. Although the structure of subsequent sleep architecture was unchanged, there was a highly significant increase in the spindle density following the learning condition compared with nonlearning (Fig. 1). This was particularly pronounced during the early part of sleep, averaging $33.5 \%$ in the first 90 min after sleep onset. Additionally, sleep spindle density was correlated positively with recall after sleep and with immediate recall tested during learning before sleep. These findings agree with observations by Meier-Koll et al. (1999), who reported a similar increase in spindles during sleep in humans following learning a maze task. Notably, Fogel et al. (2002) reported increased sleep spindle density in humans after training on a procedural pursuit rotor task. Increased spindle activity could thus represent a general sign of learning-dependent processing during sleep.

Declarative memory processing during this early period of sleep is probably also related to electrophysiological phenomena other than spindle activity. Thus, during passage into SWS in humans slow-wave rhythms, including classical delta activity $(1-4 \mathrm{~Hz})$ and slow oscillations $(<1 \mathrm{~Hz})$, increase (Fig. 2). During SWS, power in these frequency bands is maintained at a high level and only gradually decreases over time. Notably, these changes in slow oscillatory and spindle power across the initial sleep cycles are paralleled by distinct changes in the transcortical direct current (DC) potential, which shifts steeply toward negativity over frontocortical sites during transition into SWS. During SWS, this DC-potential negativity is maintained and only slightly decreases toward the end of the period (Marshall et al. 1996, 1998). The time course of DC-potential changes is strongly correlated (with average coefficients $r>0.80$ ) with changes in

\section{Learning \& Memory}




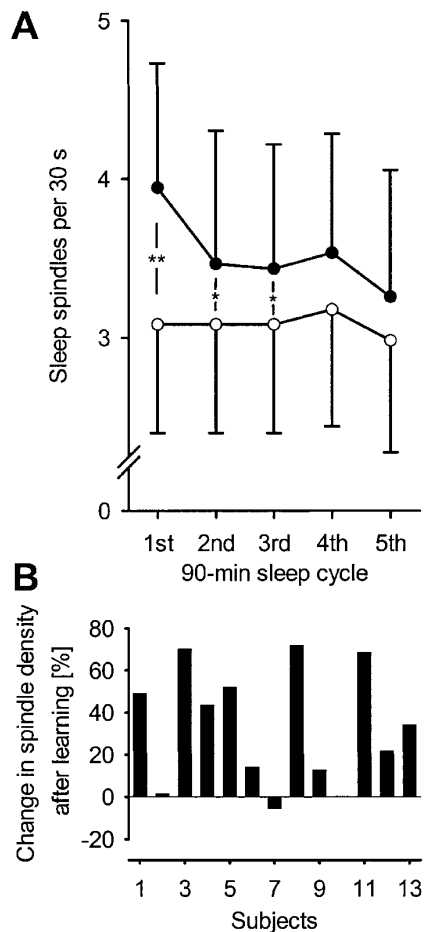

Figure 1. $(A)$ Increase in sleep spindle density (mean \pm SEM at $\mathrm{Fz}$ ) during stage 2 sleep after a period of intensive declarative learning of paired-associate wordlists (filled circles) compared with an equally strenuous nonlearning condition (open circles). Differences were compared within subjects $\left({ }^{\star} P<0.05 ;{ }^{* *} P<0.01\right)$. Large SEM values are attributable to individual differences, not differences between conditions. (B) During stage 2 sleep of the first cycle, nearly all subjects showed a substantial increase in spindle density after learning. Modified with permission from the Society for Neuroscience (c) 2002, Gais et al. 2002.

spindle, delta, and slow oscillatory activity (Marshall et al. 2003). This high correlation suggests that the generation of DCpotential negativity during SWS is closely linked with mechanisms controlling the emergence of spindle and slow oscillatory activity in thalamo-cortical feedback loops. It may reflect gradual changes in extracellular ionic concentration (e.g., due to calcium influx in cortical neurons and glial cells) resulting from the generation of spindle and slow-wave rhythms, or influences of brainstem neuromodulating systems on cortical excitability, thereby controlling the emergence of cortical spindle and slow-wave rhythms (Contreras et al. 1997b; Massimini and Amzica 2001; Amzica et al. 2002).

Although the exact source of the DC-potential negativity during SWS is presently obscure, there are now hints that it contributes to declarative memory processing during human sleep (L. Marshall, M. Mölle, M. Hallschmid, and J. Born, in prep.). That study investigated the effects of transcranial DC stimulation (tDCS) on memory formation during periods of early SWS-rich sleep (Fig. 3). During sleep after a learning period, anodal tDCS was applied. This induces widespread extracellular DC-negativity in the neocortex and was therefore expected to add to the endogenous negative DC-potential during this period. Stimulation was applied at frontocortical electrode sites intermittently over a 30-min period beginning with the onset of the first period of SWS. Compared with placebo stimulation, anodal tDCS during SWS-rich sleep distinctly increased the retention of word-pairs. This improvement is remarkable because it was found in healthy young students who were already performing at a high level on the memory task, and it was found after a SWS-rich early sleep, which per se optimizes declarative memory. tDCS did not affect procedural memory and was also ineffective if applied during a wake retention period. Notably, when applied during SWS-rich sleep, anodal tDCS increased slow oscillatory activity, suggesting that the enhancing effect of tDCS on declarative memory involves enhanced slow oscillatory EEG activity.

Slow oscillations have been associated with iterative processes of memory formation during SWS in recent concepts based largely on cortical recordings in animals (Sejnowski and Destexhe 2000; Steriade and Timofeev 2003). The slow oscillation can also be identified in the human sleep EEG, in which a spectral peak in power at $\sim 0.8 \mathrm{~Hz}$ is found (Steriade et al. 1993; Achermann and Borbély 1997; Marshall et al. 2000; Mölle et al. 2002). Slow oscillations grasp the entire thalamo-cortical system. However, they can also be recorded in isolated slabs of neocortical

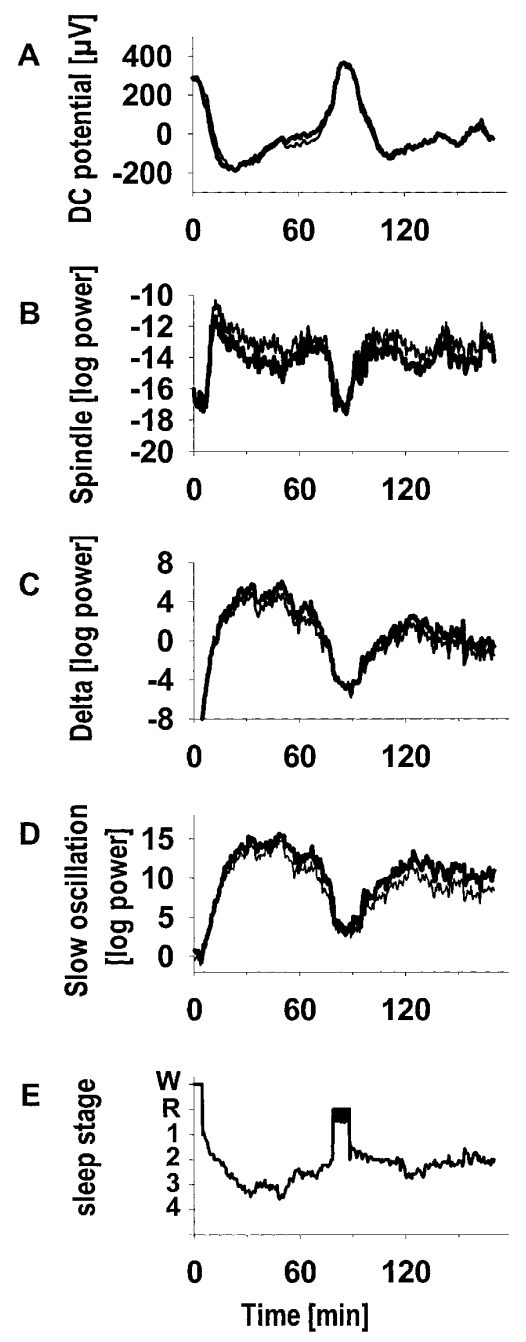

Figure 2. Changes in $(A)$ the direct current (DC) potential, in power in the $(B)$ spindle, $(C)$ delta and $(D)$ slow oscillation EEG frequency bands, and $(E)$ in sleep stages, shown for the first $3 \mathrm{~h}$ of sleep. Graphs represent means from nine subjects, time locked to sleep onset and periods of REM sleep. Note that transition into SWS is marked by a pronounced negative DC-potential shift followed by a slight decline in this negativity during SWS. This course is paralleled by strong increases in spindle, delta, and slow oscillation power at the passage into SWS followed by a slight decrease during SWS. Within individual nights, DC potential negativity and spindle as well as slow oscillatory power were strongly correlated (average $r>0.80$ ). Modified with permission from Elsevier $\odot$ 2003, Marshall et al. 2003. 
A
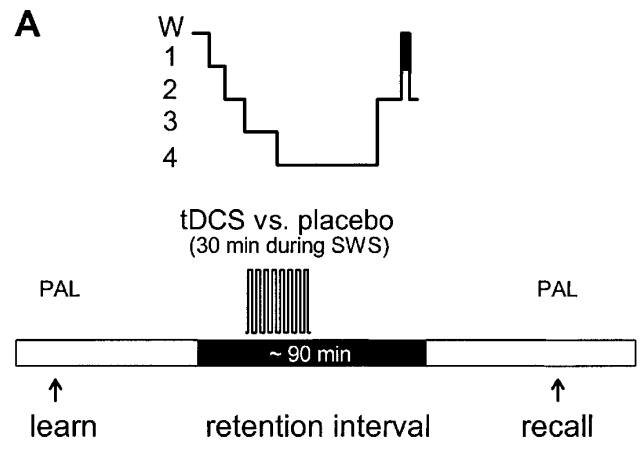

B

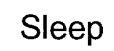

Wake

PAL
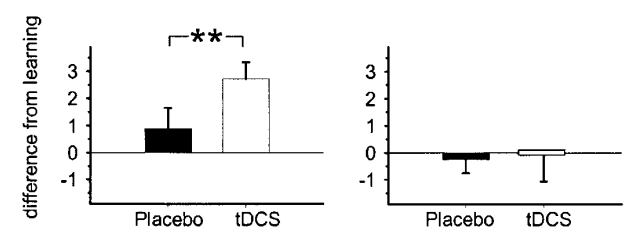

Figure 3. $(A)$ Procedure of an experiment examining the influence of transcranial direct current stimulation (tDCS) on declarative memory consolidation. The figure shows time points of learning and recall of the declarative paired associate learning (PAL) task, period of lights off (horizontal filled bar) and sleep, represented by the schematized hypnogramm (W indicates wake; 1 through 4, sleep stages 1 through 4; and vertical filled bar, REM sleep). (B) Memory performance as difference between pretest and posttest (mean \pm SEM) on the PAL task across retention periods of sleep (left) and wakefulness (right), during which either tDCS (empty bars) or placebo stimulation (filled bars) was applied. The main effect is an increase in memory retention over periods of sleep with tDCS $\left({ }^{* *} P<0.01\right)$. Modified with permission from L. Marshall, M. Mölle, M. Hallschmid, and J. Born (in prep.).

tissue, which for this reason is considered as their primary generator. In humans, the negative half-wave of the slow oscillation corresponds to a depth-positive neocortical field potential that is associated with widespread intracellular hyperpolarization (Steriade 1994; Mölle et al. 2002). Conversely, the positive half-wave marks a depth-negative extracellular field potential that reflects widespread cortical depolarization. Slow oscillations via corticothalamic fibers therefore exert a fundamental temporal grouping effect on spindle activity that is known to originate from the thalamic nucleus reticularis (Contreras and Steriade 1995; Destexhe et al. 1999). Spindle activity is stimulated during the depolarizing "up" state and suppressed during the hyperpolarizing "down" state of the slow oscillation. However, the grouping effect of slow oscillations also extends to higher frequency bands, including $\beta$ and $\gamma$ activity (Mölle et al. 2002).

There is preliminary evidence that the grouping influence of slow oscillations is also of relevance for the reprocessing of memories in humans (M. Mölle, L. Marshall, S. Gais, and J. Born, in prep.). This study used the coherence in scalp-recorded EEG activity as a correlate of the encoding of memory representations. EEG coherence is a large-scale measure that depicts the covariation in electrical activity between two distant brain regions. The synchronized activity in distributed neocortical networks, as reflected by coherence in EEG activity, can be considered to reflect the binding of different aspects of a stimulus into a uniform representation. With the encoding of complex representations as induced by written words and pictures, involving associations between different modalities, binding extends over distant brain regions and leads to increased coherence in different EEG frequency bands between distant brain regions (von Stein et al. 1999; Weiss and Rappelsberger 2000). In line with those studies, M. Mölle, L. Marshall, S. Gais, and J. Born (in prep.) observed a marked increase in EEG coherence when subjects before a period of nocturnal sleep learned a list of paired-associate words, compared with a nonlearning control condition (in which subjects were required to count the number of letters in words containing curved lines). This was reflected by the fact that the number of electrode sites between which EEG coherence was significantly higher during learning of the words than during nonlearning drastically exceeded the number of electrode sites with a reversed relation. During the early part of nocturnal sleep, there were only marginal increases in EEG coherence after learning compared with nonlearning when analysis was performed on periods of non-REM sleep in general. However, EEG coherence after learning was strikingly increased when the analysis was performed time-locked to the occurrence of slow oscillations during nonREM sleep and SWS. This analysis revealed robust learningdependent increases in coherence that emerged selectively during the depolarizing up phase of the slow oscillations and that were related to the delta and slow oscillatory as well as the spindle and $\gamma$ frequency bands. Although the learning task in this study was complex and not designed to induce a specific topographical distribution of coherences, this observation may be taken as an initial hint of a cortical reprocessing of recently encoded memories in humans and entails a special relevance for the grouping effect of slow oscillations.

In summary, the investigation of electrophysiological brain activity in humans revealed increased spindle density during early non-REM sleep and in EEG coherence during the depolarizing up state of slow oscillations after declarative learning. Because these changes followed declarative learning, a causative role of these phenomena for sleep-dependent memory consolidation remains to be established. Nevertheless, such findings agree well with recent concepts assuming that the consolidation of newly acquired declarative memories and their integration for long-term storage relies on an iterative reprocessing of these memories in hippocampo-neocortical circuitry (Buzsáki 1998; Sejnowski and Destexhe 2000; Steriade and Timofeev 2003). Central to this view is the grouping effect of slow oscillations originating in the neocortex. The long-range synchrony in cortical activity associated with the up state of slow oscillations drives thalamo-cortical spindle activity, which through the strong and simultaneous calcium influx into cortical pyramidal cells predisposes these cells toward the induction of plastic synaptic changes that underlie long-term memory formation. Concurrently, the strong synchronous cortical excitation associated with the slow oscillation up state might facilitate the occurrence of hippocampal sharp wave-ripple activity, a pattern thought to reflect reactivation of memories at the hippocampal level and their propagation to neocortical networks (Sirota et al. 2003). Slow oscillations driving coincident inputs from thalamic spindle and hippocampal sharp wave-ripple activity to cortical populations might in this way set the stage for plastic processes specifically in neocortical representations of newly encoded memories. Interestingly, miniature EPSP have been considered as one mechanism initiating the depolarizing up state of slow oscillations. These miniature potentials summate during the down state and thereby contribute to the depolarization of cortical pyramidal cells above threshold (Bazhenov et al. 2002). There is some evidence suggesting that the probability of such miniature EPSPs during the silent down phase is selectively enhanced at synapses previously activated during associative learning (Eliot et al. 1994; Oliet et al. 1996; Bao et al. 1998). If so, this would mean that slow oscillation-driven reprocessing during sleep originates preferen-

\section{Learning \& Memory}


tially in those neocortical neuron populations previously engaged in encoding during the wake phase.

\section{Acetylcholine, a neurotransmitter regulating sleep and memory}

Acetylcholine (ACh) is a neurotransmitter that has long received much attention in memory research, mainly in relation to Alzheimer's disease. It is also involved in the regulation of the nonREM/REM sleep cycle (Hobson et al. 1998). Cholinergic activation in the central nervous system mainly stems from two regions: the mesopontine tegmentum and the nucleus basalis of Meynert. The cells of the pedunculopontine and laterodorsal tegmentum are genuinely involved in the regulation of sleep stages, which is described in the reciprocal-interaction model of REM/ non-REM alternation (Pace-Schott and Hobson 2002). During wakefulness and REM sleep, these cells provide cholinergic input to thalamocortical neurons, which in turn activate the cortex via glutamatergic projections (Steriade 2003). Other projections from the tegmentum, also using glutamate, activate the nucleus basalis of Meynert, which in turn provides cholinergic activation throughout the cortex (Rasmusson et al. 1994).

The effects of ACh on memory have to be regarded separately for the acquisition, consolidation, and recall phase and for different memory systems (Olton et al. 1991). A number of studies did this by using either cholinergic receptor antagonists (e.g., scopolamine) or cholinesterase inhibitors (e.g., physostigmine). The latter increase the availability of ACh by preventing its breakdown in the synaptic cleft. The main outcome of these studies was a reduced acquisition of new memories under conditions of cholinergic deficiency, perhaps via an influence on attentional processes (Petersen 1977; Sarter et al. 2003).

According to a model of cholinergic memory modulation by Hasselmo (1999), ACh inhibits feedback loops within the hippocampus and between the hippocampus and neocortex. Thus, high cholinergic activity during wakefulness allows encoding of new declarative memories, whereas low cholinergic activity during SWS supports the spontaneous replay of newly acquired information in the hippocampus. As outlined above, this replay is thought to lead to a transfer of information from the temporary hippocampal to the permanent neocortical storage and to memory consolidation (Buzsáki 1989; Hasselmo 1999). Only very few studies have used declarative memory tasks and postlearning modulation of the cholinergic system to directly test this model. In one experiment, Rogers and Kesner (2003) had rats learn a hippocampus-dependent maze task. Before or after learning, they injected the cholinesterase inhibitor physostigmine into the hippocampal CA3 region. They conclude that "physostigmine impairs acquisition by a disruption of the consolidation process."

In a study in our own laboratory, we used a similar experimental design in humans to elucidate the role of ACh in sleepdependent consolidation (Gais and Born 2004). Subjects learned paired-associate wordlists and practiced a mirror tracing task in the evening before a 3 -h period of sleep or wakefulness during which they received an infusion of either physostigmine or placebo. Recall performance was then tested. As hypothesized, the results showed significantly impaired wordlist recall after physostigmine during sleep, whereas mirror tracing performance was not affected. Thus, the predictions of Hasselmo's (1999) model could be confirmed (Fig. 4). The inhibition of cholinergic activity during SWS plays an important role in sleep-related memory consolidation, possibly by allowing feedback loops in the hippocampus to become active. Notably, the effect of physostigmine did not depend on changes of SWS or spindle activity because it occurred also in subjects whose sleep architecture did not show obvious changes during infusion of the cholinergic agonist. Be-

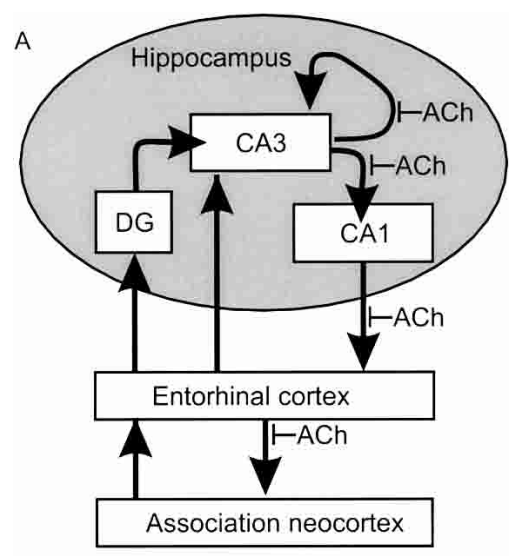

B

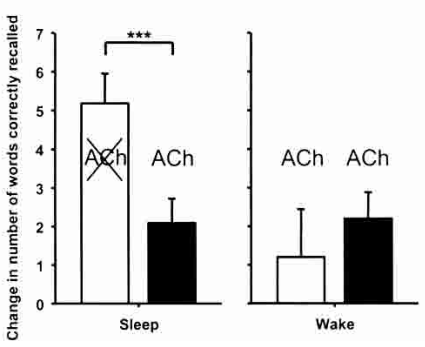

Figure 4. (A) According to a widely held model, the hippocampus temporarily stores new memories and transfers them gradually to the neocortex. Hasselmo (1999) reviews evidence that feedback pathways within the hippocampal CA3 region and from CA3 to CA1 and further to the neocortex are inhibited during wakefulness and REM sleep by acetylcholine (ACh). During SWS, these synapses become disinhibited because of the low cholinergic tone, and information can flow back to the neocortex. (B) Undisturbed SWS features low levels of ACh (left, empty bar). When during SWS-rich sleep cholinergic tone is increased by infusion of the cholinesterase inhibitor physostigmine (left, empty bar), the sleeprelated memory benefit is abolished for hippocampus-dependent, declarative wordlist memory $\left({ }^{* *} P<0.001\right)$. During wakefulness, which is accompanied by already high levels of ACh, physostigmine has no such effect (right, filled bar). Modified with permission from Elsevier ( $) 1999$, Hasselmo 1999, and with permission from The National Academy of Sciences of the United States of America $\odot$ 2004, Gais and Born 2004.

cause this oscillatory activity during SWS arises from thalamocortical networks, this finding further supports the view that physostigmine acted mainly on hippocampal memory representations in this study.

Neuroendocrine contributions to memory consolidation The neuroendocrine hypothalamic-pituitary-adrenocortical system is related to both memory formation and sleep. Cortisol (corticosterone in rats) released from the adrenal gland is the main effector of this neuroendocrine system, feeding back also to the brain. It enhances the acquisition of new and interferes with retrieval of old memories during wakefulness (de Quervain et al. 2000; Roozendaal 2000; Wolf, 2003). During the first hours of nocturnal sleep, cortisol levels drop to a minimum due to circadian rhythm and a synergistic inhibition of pituitary-adrenocortical activity by SWS (Born et al. 1988; Bierwolf et al. 1997). Concurrently, declarative memory consolidation is enhanced during SWS-rich early sleep. If, during this period of sleep, hormone levels are temporarily augmented by infusion of cortisol, the beneficial effect of sleep on memory consolidation is eradicated (Plihal and Born 1999b). Procedural memory for mirror tracing skills was not influenced by cortisol. This effect seems to be mediated by glucocorticoid receptors (Plihal et al. 1999). There is evidence that activation of glucocorticoid receptors sup- 
presses hippocampal output from CA1 neurons (de Kloet et al. 1998). This kind of effect could explain a detrimental influence of glucocorticoids on hippocampal memory reprocessing and on the propagation of the respective excitatory output to neocortical networks. Thus, one way in which sleep enhances memory consolidation seems to be by providing an optimal endocrine environment and preventing hormonal feedback signals from interfering with hippocampal memory processing. Other hormonal systems, such as the somatotropic axis with its release of growth hormone, also show distinct changes in their secretory activity during sleep and might also contribute toward memory consolidation, but remain yet to be studied.

\section{Conclusion}

Together, these experiments present evidence that sleep provides special conditions enhancing declarative memory consolidation, which in turn allow memory traces to be actively reprocessed and strengthened during sleep. This reprocessing might also include a transfer of information temporarily stored in the hippocampus to neocortical brain regions, and a stabilization or enhancement of synaptic connections. However, a qualitative reprocessing of information during sleep might also occur when during this process newly acquired memories become integrated with long-term memories assumed to reside in neocortical networks (McClelland et al. 1995). In a recent study, Wagner et al. (2004) had subjects do a simple task that had one obvious (and long) and one hidden (and much shorter) solution. Thus, subjects finding the hidden solution could greatly shorten the time needed to complete the task. Subjects were confronted with the task for a short period in the evening before an interval of sleep or wakefulness. When subjects were confronted with the task again in the morning, those of them having slept were significantly better in finding the hidden rule than those who had not. They were also significantly better than were subjects who slept during the night but were not confronted with the task before, ruling out effects of fatigue and sleepiness. A possible interpretation of these data is that the representation of the task built before sleep is reprocessed during sleep and "restructured" in a way that allows the new solution to be found.

Regarding the ever more complex data on the association between sleep and memory, all monocausal models attributing effects to one mechanism are likely to fail, especially if they use descriptive concepts such as "REM sleep" that represent an accumulation of many different physiological processes occurring simultaneously. Here, we have roughly outlined some of the physiological mechanisms that may contribute to the enhancement of declarative memory in an off-line process taking place during human sleep. Of course, it is far from being well-established that these mechanisms play a causative role for declarative memory consolidation during sleep. However, it is precisely this question that should stimulate further testing in a way that also takes into account that not just one but several mechanisms during the different sleep states act in concert to consolidate memory.

\section{References}

Achermann, P. and Borbély, A.A. 1997. Low-frequency $(<1 \mathrm{~Hz})$ oscillations in the human sleep electroencephalogram. Neuroscience 81: 213-222.

Amzica, F., Massimini, M., and Manfridi, A. 2002. Spatial buffering during slow and paroxysmal sleep oscillations in cortical networks of glial cells in vivo. J. Neurosci. 22: 1042-1053.

Aserinsky, E. and Kleitman, N. 1953. Regularly occurring periods of eye motility, and concomitant phenomena, during sleep. Science 118: 273-274.

Bao, J.X., Kandel, E.R., and Hawkins, R.D. 1998. Involvement of presynaptic and postsynaptic mechanisms in a cellular analog of classical conditioning at aplysia sensory-motor neuron synapses in isolated cell culture. J. Neurosci. 18: 458-466.
Bazhenov, M., Timofeev, I., Steriade, M., and Sejnowski, T.J. 2002. Model of thalamocortical slow-wave sleep oscillations and transitions to activated states. J. Neurosci. 22: 8691-8704.

Bierwolf, C., Struve, K., Marshall, L., Born, J., and Fehm, H.L. 1997. Slow wave sleep drives inhibition of pituitary-adrenal secretion in humans. J. Neuroendocrinol. 9: 479-484.

Born, J. and Gais, S. 2000. REM sleep deprivation: The wrong paradigm leading to wrong conclusions. Behav. Brain Sci. 23: 912-913.

Born, J., Muth, S., and Fehm, H.L. 1988. The significance of sleep onset and slow wave sleep for nocturnal release of growth hormone $(\mathrm{GH})$ and cortisol. Psychoneuroendocrinology 13: 233-243.

Buzsáki, G. 1989. Two-stage model of memory trace formation: A role for "noisy" brain states. Neuroscience 31: 551-570.

. 1996. The hippocampo-neocortical dialogue. Cereb. Cortex 6: $81-92$.

. 1998. Memory consolidation during sleep: A neurophysiological perspective. J. Sleep Res. 7: 17-23.

Chernik, D.A. 1972. Effect of REM sleep deprivation on learning and recall by humans. Percept. Mot. Skills 34: 283-294.

Contreras, D. and Steriade, M. 1995. Cellular basis of EEG slow rhythms: A study of dynamic corticothalamic relationships. J. Neurosci. 15: 604-622.

Contreras, D., Destexhe, A., Sejnowski, T.J., and Steriade, M. 1997a. Spatiotemporal patterns of spindle oscillations in cortex and thalamus. J. Neurosci. 17: 1179-1196.

Contreras, D., Destexhe, A., and Steriade, M. 1997b. Intracellular and computational characterization of the intracortical inhibitory control of synchronized thalamic inputs in vivo. J. Neurophysiol. 78: $335-350$.

de Kloet, E.R., Vreugdenhil, E., Oitzl, M.S., and Joels, M. 1998. Brain corticosteroid receptor balance in health and disease. Endocr. Rev. 19: 269-301.

de Quervain, D.J., Roozendaal, B., Nitsch, R.M., McGaugh, J.L., and Hock, C. 2000. Acute cortisone administration impairs retrieval of long-term declarative memory in humans. Nat. Neurosci. 3: $313-314$

Destexhe, A., Contreras, D., and Steriade, M. 1999. Cortically-induced coherence of a thalamic-generated oscillation. Neuroscience 92: $427-443$.

Eliot, L.S., Kandel, E.R., and Hawkins, R.D. 1994. Modulation of spontaneous transmitter release during depression and posttetanic potentiation of aplysia sensory-motor neuron synapses isolated in culture. J. Neurosci. 14: 3280-3292.

Empson, J.A. and Clarke, P.R. 1970. Rapid eye movements and remembering. Nature 227: 287-288.

Feldman, R. and Dement, W. 1968. Possible relationships between REM sleep and memory consolidation. Psychophysiology 5: 243-251.

Fischer, S., Hallschmid, M., Elsner, A.L., and Born, J. 2002. Sleep forms memory for finger skills. Proc. Natl. Acad. Sci. 99: 11987-11991.

Fogel, S.M., Jacob, J., and Smith, C.T. 2002. The role of sleep spindles in simple motor procedural learning. Sleep 25: A279-A280.

Fowler, M.J., Sullivan, M.J., and Ekstrand, B.R. 1973. Sleep and memory. Science 179: 302-304.

Gais, S. and Born, J. 2004. Low acetylcholine during slow-wave sleep is critical for declarative memory consolidation. Proc. Natl. Acad. Sci. 101: $2140-2144$.

Gais, S., Plihal, W., Wagner, U., and Born, J. 2000. Early sleep triggers memory for early visual discrimination skills. Nat. Neurosci. 3: $1335-1339$

Gais, S., Mölle, M., Helms, K., and Born, J. 2002. Learning-dependent increases in sleep spindle density. J. Neurosci. 22: 6830-6834.

Hasselmo, M.E. 1999. Neuromodulation: Acetylcholine and memory consolidation. Trends Cogn. Sci. 3: 351-359.

Hobson, J.A., Stickgold, R., and Pace-Schott, E.F. 1998. The neuropsychology of REM sleep dreaming. Neuroreport 9: R1-R14.

Jenkins, J.G. and Dallenbach, K.M. 1924. Obliviscence during sleep and waking. Am. J. Psychol. 35: 605-612.

Karni, A., Tanne, D., Rubenstein, B.S., Askenasy, J.J., and Sagi, D. 1994 Dependence on REM sleep of overnight improvement of a perceptual skill. Science 265: 679-682.

Kudrimoti, H.S., Barnes, C.A., and McNaughton, B.L. 1999. Reactivation of hippocampal cell assemblies: Effects of behavioral state, experience, and EEG dynamics. J. Neurosci. 19: 4090-4101.

Lewin, I. and Glaubman, H. 1975. The effect of REM deprivation: Is it detrimental, beneficial, or neutral? Psychophysiology 12: 349-353.

Louie, K. and Wilson, M.A. 2001. Temporally structured replay of awake hippocampal ensemble activity during rapid eye movement sleep. Neuron 29: 145-156.

Maquet, P. 2001. The role of sleep in learning and memory. Science 294: 1048-1052.

Maquet, P., Laureys, S., Peigneux, P., Fuchs, S., Petiau, C., Phillips, C., Aerts, J., Del Fiore, G., Degueldre, C., Meulemans, T., et al. 2000.

\section{Learning \& Memory}


Experience-dependent changes in cerebral activation during human REM sleep. Nat. Neurosci. 3: 831-836.

Marshall, L., Mölle, M., Michaelsen, S., Fehm, H.L., and Born, J. 1996. Slow potential shifts at sleep-wake transitions and shifts between NREM and REM sleep. Sleep 19: 145-151.

Marshall, L., Mölle, M., Fehm, H.L., and Born, J. 1998. Scalp recorded direct current brain potentials during human sleep. Eur. J. Neurosci. 10: $1167-1178$.

. 2000. Changes in direct current (DC) potentials and infra-slow EEG oscillations at the onset of the luteinizing hormone (LH) pulse. Eur. J. Neurosci. 12: 3935-3943.

Marshall, L., Mölle, M., and Born, J. 2003. Spindle and slow wave rhythms at slow wave sleep transitions are linked to strong shifts in the cortical direct current potential. Neuroscience 121: 1047-1053.

Massimini, M. and Amzica, F. 2001. Extracellular calcium fluctuations and intracellular potentials in the cortex during the slow sleep oscillation. J. Neurophysiol. 85: 1346-1350.

McClelland, J.L., McNaughton, B.L., and O'Reilly, R.C. 1995. Why there are complementary learning systems in the hippocampus and neocortex: Insights from the successes and failures of connectionist models of learning and memory. Psychol. Rev. 102: 419-457.

McNaughton, B.L., Barnes, C.A., Battaglia, F.P., Bower, M.R., Cowen, S.L., Ekstrom, A.D., Gerrard, J.L., Hoffman, K.L., Houston, F.P., Karten, Y., et al. 2003. Off-line reprocessing of recent memory and its role in memory consolidation: A progress report. In Sleep and brain plasticity (ed. P. Maquet, et al.), pp. 225-246. Oxford University Press, New York.

Meier-Koll, A., Bussmann, B., Schmidt, C., and Neuschwander, D. 1999. Walking through a maze alters the architecture of sleep. Percept. Mot. Skills 88: 1141-1159.

Mölle, M., Marshall, L., Gais, S., and Born, J. 2002. Grouping of spindle activity during slow oscillations in human non-rapid eye movement sleep. J. Neurosci. 22: 10941-10947.

Newman, E.B. 1939. Forgetting of meaningful material during sleep and waking. Am. J. Psychol. 52: 65-71.

Oliet, S.H., Malenka, R.C., and Nicoll, R.A. 1996. Bidirectional control of quantal size by synaptic activity in the hippocampus. Science 271: $1294-1297$.

Olton, D., Markowska, A., Voytko, M.L., Givens, B., Gorman, L., and Wenk, G. 1991. Basal forebrain cholinergic system: A functional analysis. Adv. Exp. Med. Biol. 295: 353-372.

Pace-Schott, E.F. and Hobson, J.A. 2002. The neurobiology of sleep: Genetics, cellular physiology and subcortical networks. Nat. Rev. Neurosci. 3: 591-605.

Pavlides, C. and Winson, J. 1989. Influences of hippocampal place cell firing in the awake state on the activity of these cells during subsequent sleep episodes. J. Neurosci. 9: 2907-2918.

Petersen, R.C. 1977. Scopolamine induced learning failures in man. Psychopharmacology (Berl) 52: 283-289.

Plihal, W. and Born, J. 1997. Effects of early and late nocturnal sleep on declarative and procedural memory. J. Cogn. Neurosci. 9: 534-547.

. 1999a. Effects of early and late nocturnal sleep on priming and spatial memory. Psychophysiology 36: 571-582.

. 1999b. Memory consolidation in human sleep depends on inhibition of glucocorticoid release. Neuroreport 10: 2741-2747.

Plihal, W., Pietrowsky, R., and Born, J. 1999. Dexamethasone blocks sleep induced improvement of declarative memory. Psychoneuroendocrinology 24: 313-331.

Qin, Y.L., McNaughton, B.L., Skaggs, W.E., and Barnes, C.A. 1997. Memory reprocessing in corticocortical and hippocampocortical neuronal ensembles. Philos. Trans. R. Soc. Lond B 352: 1525-1533.

Rasmusson, D.D., Clow, K., and Szerb, J.C. 1994. Modification of neocortical acetylcholine release and electroencephalogram desynchronization due to brainstem stimulation by drugs applied to the basal forebrain. Neuroscience 60: 665-677.

Ribeiro, S., Gervasoni, D., Soares, E.S., Zhou, Y., Lin, S.C., Pantoja, J,. Lavine, M., and Nicolelis, M.A. 2004. Long-lasting novelty-induced neuronal reverberation during slow-wave sleep in multiple forebrain areas. PLoS. Biol. 2: E24.
Rogers, J.L. and Kesner, R.P. 2003. Cholinergic modulation of the hippocampus during encoding and retrieval. Neurobiol. Learn. Mem. 80: $332-342$.

Roozendaal, B. 2000. 1999 Curt P. Richter award: Glucocorticoids and the regulation of memory consolidation. Psychoneuroendocrinology 25: $213-238$.

Sarter, M., Bruno, J.P., and Givens, B. 2003. Attentional functions of cortical cholinergic inputs: What does it mean for learning and memory? Neurobiol. Learn. Mem. 80: 245-256.

Sejnowski, T.J. and Destexhe, A. 2000. Why do we sleep? Brain Res. 886: $208-223$

Siapas, A.G. and Wilson, M.A. 1998. Coordinated interactions between hippocampal ripples and cortical spindles during slow-wave sleep. Neuron 21: 1123-1128.

Siegel, J.M. 2001. The REM sleep-memory consolidation hypothesis. Science 294: 1058-1063.

Sirota, A., Csicsvari, J., Buhl, D., and Buzsáki, G. 2003. Communication between neocortex and hippocampus during sleep in rodents. Proc. Natl. Acad. Sci. 100: 2065-2069.

Smith, C. and MacNeill, C. 1994. Impaired motor memory for a pursuit rotor task following stage 2 sleep loss in college students. J. Sleep Res. 3: 206-213.

Smith, C.T. and Fazekas, A. 1997. Amounts of REM sleep and stage 2 required for efficient learning. Sleep 26: 690.

Steriade, M. 1994. Sleep oscillations and their blockage by activating systems. J. Psychiatry Neurosci. 19: 354-358.

. 2003. The corticothalamic system in sleep. Front Biosci. 8: d878-d899.

Steriade, M. and Timofeev, I. 2003. Neuronal plasticity in thalamocortical networks during sleep and waking oscillations. Neuron 37: 563-576.

Steriade, M., Nunez, A., and Amzica, F. 1993. A novel slow $(<1 \mathrm{~Hz})$ oscillation of neocortical neurons in vivo: Depolarizing and hyperpolarizing components. J. Neurosci. 13: 3252-3265.

Stickgold, R., James, L., and Hobson, J.A. 2000a. Visual discrimination learning requires sleep after training. Nat. Neurosci. 3: 1237-1238.

Stickgold, R., Whidbee, D., Schirmer, B., Patel, V., and Hobson, J.A. 2000b. Visual discrimination task improvement: A multi-step process occurring during sleep. J. Cogn. Neurosci. 12: 246-254.

Stickgold, R., Hobson, J.A., Fosse, R., and Fosse, M. 2001. Sleep, learning, and dreams: Off-line memory reprocessing. Science 294: $1052-1057$.

Van Ormer, E.B. 1933. Sleep and retention. Psychol. Bull. 30: 415-439.

Vertes, R.P. and Eastman, K.E. 2000. The case against memory consolidation in REM sleep. Behav. Brain Sci. 23: 867-876.

von Stein, A., Rappelsberger, P., Sarnthein, J., and Petsche, H. 1999. Synchronization between temporal and parietal cortex during multimodal object processing in man. Cereb. Cortex 9: 137-150.

Wagner, U., Gais, S., and Born, J. 2001. Emotional memory formation is enhanced across sleep intervals with high amounts of rapid eye movement sleep. Learn. Mem. 8: 112-119.

Wagner, U., Fischer, S., and Born, J. 2002. Changes in emotional responses to aversive pictures across periods rich in slow-wave sleep versus rapid eye movement sleep. Psychosom. Med. 64: 627-634.

Wagner, U., Gais, S., Haider, H., Verleger, R., and Born, J. 2004. Sleep inspires insight. Nature 427: 352-355.

Walker, M.P., Brakefield, T., Morgan, A., Hobson, J.A., and Stickgold, R. 2002. Practice with sleep makes perfect: Sleep-dependent motor skill learning. Neuron 35: 205-211.

Weiss, S. and Rappelsberger, P. 2000. Long-range EEG synchronization during word encoding correlates with successful memory performance. Brain Res. Cogn Brain Res. 9: 299-312.

Wilson, M.A. and McNaughton, B.L. 1994. Reactivation of hippocampal ensemble memories during sleep. Science 265: 676-679.

Wolf, O.T. 2003. HPA axis and memory. Best. Pract. Res. Clin. Endocrinol. Metab. 17: 287-299.

Yaroush, R., Sullivan, M.J., and Ekstrand, B.R. 1971. Effect of sleep on memory, II: Differential effect of the first and second half of the night. J. Exp. Psychol. 88: 361-366. 


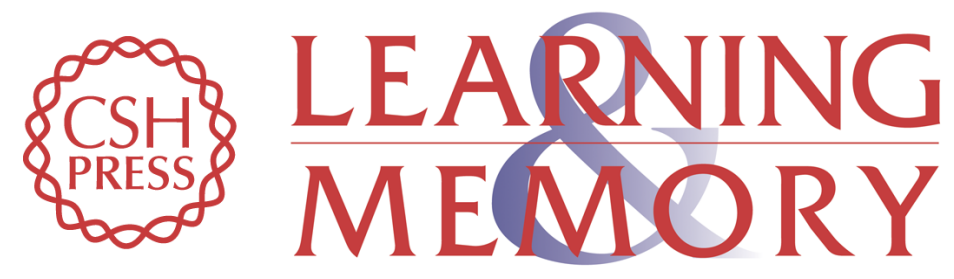

\section{Declarative memory consolidation: Mechanisms acting during human sleep}

Steffen Gais and Jan Born

Learn. Mem. 2004, 11:

Access the most recent version at doi:10.1101/lm.80504

References This article cites 83 articles, 24 of which can be accessed free at: http://learnmem.cshlp.org/content/11/6/679.full.html\#ref-list-1

License

Email Alerting Receive free email alerts when new articles cite this article - sign up in the box at the Service top right corner of the article or click here. 